Daniel Rico T. De Jesus, MD

Patrick Joseph L. Estolano, MD

Department of Otorhinolaryngology Head and Neck Surgery

Jose R. Reyes Memorial Medical Center
Correspondence: Dr. Patrick Joseph L. Estolano

Department of Otorhinolaryngology

Head and Neck Surgery

Jose R. Reyes Memorial Medical Center

San Lazaro Compound, Rizal Avenue

Sta. Cruz, Manila 1003

Philippines

Phone: +639175280334 / (632) 87119491 local 320

E-mail: patrick_esto@yahoo.com

The authors declared that this presents original material that is not being considered for publication or has not been published or accepted for publication elsewhere or in part, in print or electronic media; that the requirements for authorship have been met by all the authors, and the author believes that the manuscript represents honest work.

Disclosures: The authors signed disclosures that there are no financial or other (including personal) relationship, intellectual passion, political or religious beliefs, and institutional affiliations that might led to conflict of interest.

Presented at the Philippine Society of Otolaryngology- Head and Neck Surgery 1st Virtual Interesting Case Contest (15tace), July 22, 2020 and at the Annual Interdepartmental Research Forum of the Jose R. Reyes Memorial Medical Center. December 4, 2019. Jose R. Reyes Memorial Medical Center, Manila City.

\title{
Blindness from Fungal Rhinosinusitis of the Paranasal Sinuses: A Case Report
}

\section{ABSTRACT}

Objective: To present a unique case of blindness resulting from fungal rhinosinusitis involving multiple sinuses mimicking a malignant process in a pregnant patient.

\section{Methods:}

\author{
Design: Case Report \\ Setting: Tertiary Government Training Hospital \\ Patient: One
}

Result: A 36-year-old pregnant woman developed unilateral blindness during her $20^{\text {th }}$ week of gestation with a history of binocular diplopia, unilateral nasal obstruction and anosmia for 13 months during the pre-pregnancy period. Sphenoid sinus malignancy was suspected on imaging. The planned biopsy was intraoperatively shifted to endoscopic sinus surgery when clay-like materials were seen involving the left maxillary sinus and bilateral sphenoid and ethmoid sinuses. Histopathologic examination confirmed fungal growth. Postoperatively, nasal symptoms resolved but blindness of the left eye and blurring of vision of the right eye persisted.

Conclusion: Fungal rhinosinusitis rarely occurs in multiple sinuses and is commonly misdiagnosed. It can afflict pregnant patients and mimic a malignant process. A high index of suspicion early on, especially in the presence of nasal congestion and diplopia may prevent potentially irreversible complications.

\section{Keywords: sinusitis; blindness; sphenoid sinus; maxillary sinus; pregnancy}

Fungal rhinosinusitis is a rarely diagnosed disease affecting $12,000,000$ globally $^{1}$ with an incidence of 5.4 and $8.2 \%$ in South Korea and Japan, respectively. ${ }^{2}$ Sinus fungus ball is a noninvasive type that is predominantly unilateral in presentation and grows in wet, moist, cavities of the paranasal sinuses, mostly in females and elderly hosts with normal immunologic status. ${ }^{3}$ Since symptoms are non-specific, its presentation is indistinguishable from usual chronic bacterial rhinosinusitis and may only be discovered incidentally. ${ }^{4}$ In some cases, it may even mimic a malignant tumor especially when the orbit is involved. ${ }^{5}$ We present the insidious development of common non-specific nasal symptoms towards blindness in a pregnant patient with sinus fungus ball. 


\section{CASE REPORT}

A 36-year-old G4P2 (2-0-1-2) woman presented with a 13-month history of binocular diplopia and gradually progressing to blurring of vision. She also complained of occasional headache, left nasal obstruction and anosmia. No nasal discharge, epistaxis or difficulty of breathing were noted. She had an unremarkable past medical history and lived in a small apartment home with poor ventilation.

Her worsening blurring of vision prompted her to consult. A plain Cranial Computed Tomography (CT) scan revealed a lobulated soft tissue mass with coarse calcifications occupying the clivus, sphenoid, posterior ethmoid, sellar and suprasellar region (Figure 1A) and left maxillary sinus (Figure 1B) with primary consideration of a pituitary neoplasm. She was unable to obtain the further workups requested.

She was admitted under the Neurology service on her $20^{\text {th }}$ week of gestation due to left eye blindness and severe headaches (VAS score 8/10). Ophthalmologic examination revealed absent light perception in the left eye and hand perception in the right eye. Both eyes were medially deviated with lateral rectus palsy more noticeable on the left. Fundoscopic examination revealed pale optic disc with no papilledema on the left eye. An MRI of the head with diffusion weighted imaging revealed bony expansion and thinning of bilateral sphenoid sinuses and the left maxillary sinus, heterogeneously-enhancing foci occupying the nasal area displacing the sella, pituitary gland, optic chiasm and nerves superiorly hence a suspicion of sphenoid sinus malignancy was entertained. (Figure 2A, $B$ )

She was referred to ear, nose, throat - head and neck surgery (ENT-HNS) for evaluation of the suspected paranasal sinus mass. Physical examination revealed left nasal congestion with clear watery discharge on anterior rhinoscopy, and facial tenderness over the left maxillary area on palpation. Nasal videoendoscopy showed a suspicious nasopharyngeal bulge but punch biopsy revealed chronic inflammation only. Hence, maxillary antrostomy with exploration of the other sinuses via endoscopic sinus surgery was done. (Figure $3 A$ ) Intraoperative findings showed clay-like material filling the left maxillary sinus, bilateral ethmoid and sphenoid sinuses. (Figure 3B, C, D) Hematoxylin-Eosin and Gomori- Methenamine staining confirmed fungal growth of the acquired specimen. (Figures $4 A$ and $4 B$, respectively). Serum galactomannan to determine the specific organism involved was not facilitated due to financial constraints. Both mother and fetus tolerated the procedure well. Antifungal medications were not given post-operatively due to pregnancy considerations.

Post-operatively, nasal congestion, facial tenderness, headache and anosmia resolved. Ophthalmologic examination revealed no
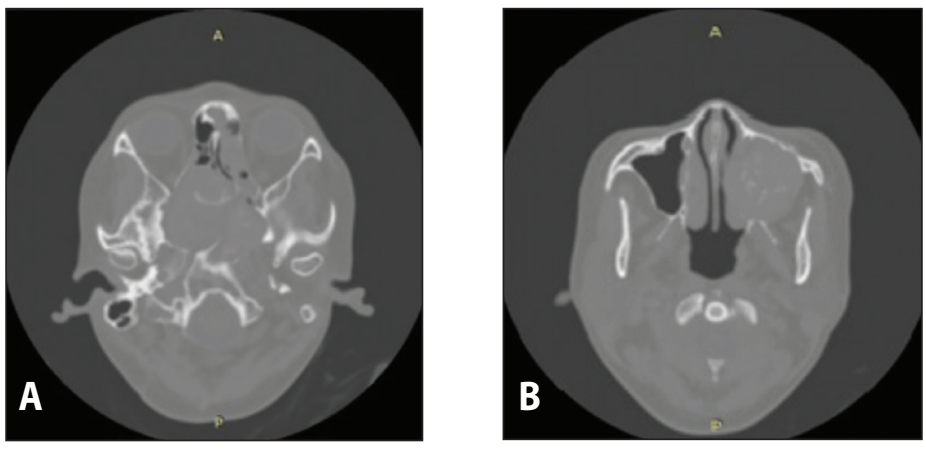

Figure 1. Plain cranial CT scan, Axial view: A. Lobulated soft tissue mass with coarse calcification occupying the clivus, sphenoid, posterior ethmoid, sellar and suprasellar region; B. Lobulated soft tissue mass occupying the left maxillary sinus.
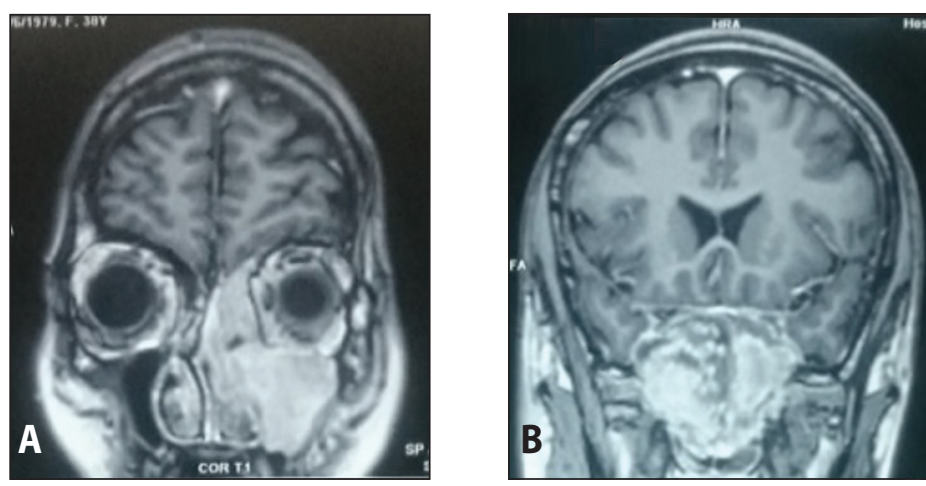

Figure 2. MRI of the head with DWI and contrast: A. Heterogeneously enhancing foci with bony expansion and thinning at the level of the left maxillary sinus; B. at the level of the sphenoid sinuses displacing the parasellar structures.
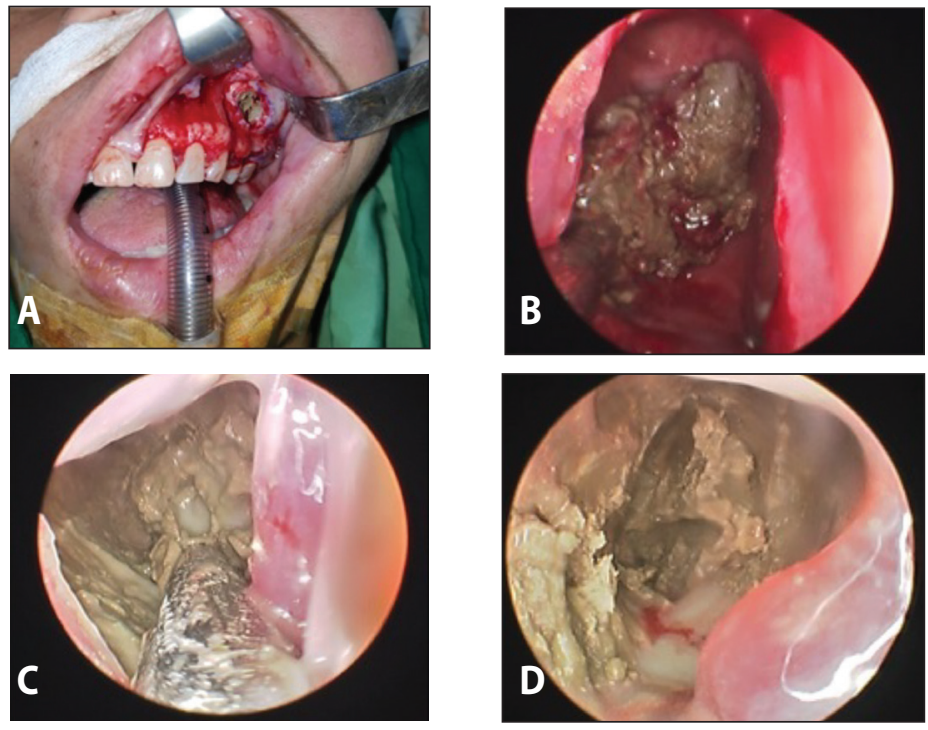

Figure 3. Intraoperative findings: A. Left maxillary sinus showing fungus ball upon opening of the anterior maxillary wall accessed thru gingivobuccal incision; and endoscopic views showing aggregates of fungus ball in the B. Left maxillary sinus, C. Sphenoid sinus and; D. Ethmoid sinus. 


\section{CASE REPORTS}
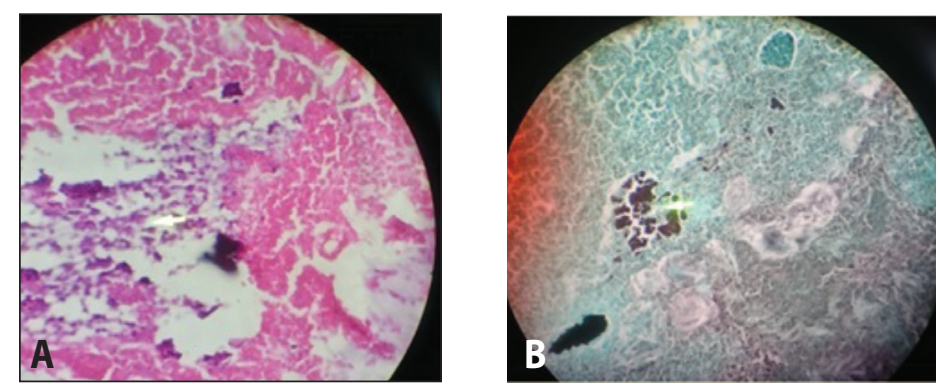

Figure 4. Histopathologic slides: A. Hematoxylin and Eosin stains showing fungal elements on high power view (white arrow) B. Histopathologic slides using Gomori methenamine staining showing collection of fungal elements in low power view (arrow).

improvement of her vision. She was discharged home with no other complications. On follow-up, she had delivered her baby at term via normal spontaneous delivery with no feto-maternal complications.

\section{DISCUSSION}

As shown in our case, blindness can be an unfortunate sequela of fungus ball. A MEDLINE (PubMed) search using the keywords "fungus ball" and "blindness" revealed a report by Kim et al. on a patient with blindness secondary to a sphenoid sinus fungus ball. ${ }^{6}$ Using the same keywords, a search of HERDIN Plus, the ASEAN Citation Index, Global Index Medicus ( Western Pacific Region Index Medicus, WPRIM and Index Medicus of the South East Asia Region, IMSEAR), Directory of Open Access Journals (DOAJ) and Google Scholar yielded no other similar cases published locally.

The most common organisms causing fungus ball are Aspergillus fumigatus, Aspergillus flavus, Alternaria, and Mucor species.' They are usually found in just one sinus; the maxillary sinus as the most frequently involved (94\%) followed by the sphenoid sinus (4-8\%).? The ethmoid sinus (3\%) is most often a continuous involvement from the maxillary sinus.? Fungal rhinosinusitis rarely occurs in multiple sinuses and is commonly misdiagnosed.?

Mostafa et al. associated a small apartment floor, poor air conditioning, and exposure to dust and cockroaches with higher incidence of fungal rhinosinusitis due to humid environment and less exposure to sunlight. ${ }^{8}$ Our patient lived in similar circumstances with no other comorbidities that could lead to her condition.

Fungal rhinosinusitis has a non-specific presentation which may lead to confusion in diagnosis. In our case, an initial impression of a nasopharyngeal tumor was considered due to the nasal endoscopic findings of a nasopharyngeal bulge and the patient's diplopia. In about $57 \%$ of patients with nasopharyngeal carcinoma and diplopia, it was observed that the infiltrating nature of the mass to extend intracranially could have involved cranial nerve $\mathrm{VI} .{ }^{9}$

In general, the visual disturbance in compressive lesions similar to our patient is attributed to the interruption of the blood supply to the optic nerve with secondary ischemic optic atrophy, as confirmed by the fundoscopic findings of a pale optic disc. ${ }^{10}$ It has been posited that accumulation of viscous eosinophilic mucin by nasal congestion can cause obstruction of sinus outflow tracts, which then allows inflammatory mediators to cause gradual sinus expansion and bony erosion." Hence, the bilateral bony expansion of the sphenoid sinus seen on MRI may have caused compression on the optic nerve, supported by the yellow-brownish friable fungus ball seen intraoperatively.

Definitive treatment for fungal rhinosinusitis involves restoration of normal sinus drainage by removal of the fungus ball, extirpation of allergic mucin, and long-term nasal steroids to prevent recurrence. ${ }^{11}$ Antifungal medications were no longer initiated as they have not been proven to be beneficial in treatment ${ }^{11}$ and could place the pregnancy and fetus at risk.

In conclusion, our case has shown that fungal rhinosinusitis can afflict a pregnant patient with non-specific nasal symptoms and ophthalmic complications mimicking a malignant process. Multiple sinus involvement of fungal rhinosinusitis, although rare, should also be considered as a differential diagnosis even in pregnant patients. A high index of suspicion early on, especially in the presence of nasal congestion and diplopia may prevent potentially irreversible complications.

\section{ACKNOWLEDGEMENTS}

We thank Dr. Gil M. Vicente and Dr. Alexander S. Dy who served as scientific advisors for this report.

\section{REFERENCES}

1. Bongomin F, Gago S, Oladele R, Denning D. Global and multi-national prevalence of fungal diseases-estimate precision. J Fungi (Basel). 2017 Oct 18;3(4):57. DOI:10.3390/jof3040057. PubMed PMID: 29371573; PubMed Central PMCID: PMC5753159.

2. J S Kim, S S So, S H Kwon' The Increasing Incidence of Paranasal Sinus Fungus Ball: A Retrospective Cohort Study in Two Hundred Forty-Five Patients for Fifteen Years. Clin Otolaryngol. 2017 Feb;42(1):175-179. DOI: 10.1111/coa.12588. PubMed PMID: 26576036.

3. Jiang R-S, Huang W-C, Liang K-L. Characteristics of sinus fungus ball: $A$ unique form of rhinosinusitis. Clin Med Insights Ear Nose Throat. 2018 Aug 3; 11:1179550618792254. DOI:10.1177/1179550618792254; PubMed PMID: 30090023; PubMed Central PMCID: PMC6077877.

4. Bhowmik B. Fungal sinusitis clinical presentation, latest management: A Review Update. Journal of Science Foundation. 2014 Jan;12(1):1728-7855. DOI: https://doi.org/10.3329/jsf.v12i1.23460.

5. Ferguson B, Lee S. Fungal Rhinosinusitis. In: Flint P, Haughey B, Lund V, Niparko J, Robbins K Regan Thomas J. et al., editors. Cummings Otolaryngology Head and neck surgery. $6^{\text {th }}$ ed. London: Saunders; 2014. p.735-736.

6. Kim JS, Kim BK, Hong SD, Kim HJ, Kim HY. Clinical characteristics of sphenoid sinus fungal ball patients with visual disturbance. Clin Exp Otorhinolaryngol. 2016 Dec;9(4):326-331. DOl: 10.21053/ceo.2015.01571. Epub 2016 May 3. PubMed PMID: 27136367; PubMed Central PMCID: PMC5115146.

7. Grosjean $P$, Weber R. Fungus balls of the paranasal sinuses: a review. Eur Arch Otorhinolaryngol. 2007 May; 264(5):461-70. DOI: 10.1007/s00405-007-0281-5; PubMed PMID: 17361410

8. Mostafa BE, El Sharnoubi MMK, El-Sersy HAA, Mahmoud MSM. Environmental risk factors in patients with noninvasive fungal sinusitis. Scientifica (Cairo). 2016;2016:5491694. DOI: 10.1155/2016/5491694; PubMed PMID: 27274885; PubMed Central PMCID: PMC4870367.

9. Ilhan $\mathrm{O}$, Sener EC, Ozyar E. Outcome of abducens nerve paralysis in patients with nasopharyngeal carcinoma. Eur J Ophthalmol. 2002 Jan-Feb; 12(1):55-9. PubMed PMID: 11936446.

10. Goldenberg-Cohen N, Ehrenberg M, Toledano H, Kornreich L, Snir M, Yassur I, Michowiz $\mathrm{S}$. Preoperative visual loss is the main cause of irreversible poor vision in children with a brain tumor. Front Neurol. 2011 Sep 30; 2:62. DOI: 10.3389/fneur.2011.00062; PubMed PMID: 21994502; PubMed Central PMCID: PMC3183350.

11. Gorovoy IR, Kazanjian M, Kersten RC, Kim HJ, Vagefi MR. Fungal rhinosinusitis and imaging modalities. Saudi J Ophthalmol. 2012 Oct; 26(4):419-26. DOI: 10.1016/j.sjopt.2012.08.009 PubMed PMID: 23961027; PubMed Central PMCID: PMC3729552. 